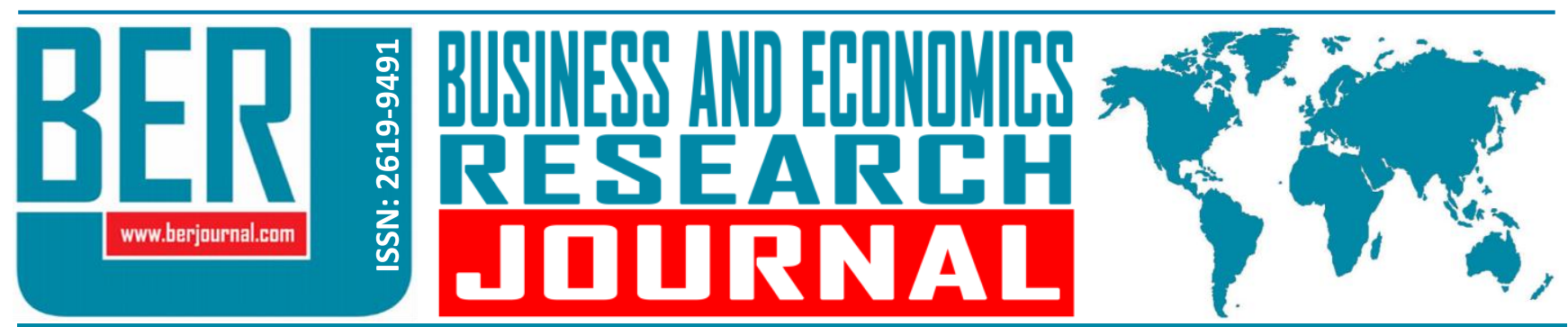

Business and Economics Research Journal Vol. 10, No. 5, 2019, pp. 1167-1178 doi: 10.20409/berj.2019.228

\title{
The Effect of Emotional Exhaustion on Workplace Ostracism and Job Insecurity in North Cyprus Hotel Industry ${ }^{1}$
}

\author{
Mehmet Necati Cizreliogullari ${ }^{\mathrm{a}}$, Ozlem Altun ${ }^{\mathrm{b}}$, Pinar Barut ${ }^{\mathrm{c}}$
}

Abstract: The aim of this study is to investigate the mediating effects of employee emotional exhaustion (EE) on workplace ostracism (WO) and job insecurity (JI) using the theory of resource conservation. In this research, structural equality modeling was conducted through 211 questionnaires, working in Hotels in Northern Cyprus. For data analysis used Microsoft Excel and Statistical Software Programs including SPSS and AMOS. The study results show that the gradual convergence of emotional exhaustion due to incapacity can lead to an increase in negative effects on job insecurity or ostracism in the workplace which gradually increases over time, impacting negatively on and ultimately increasing workplace ostracism. Although, the studies on exhaustion and insecurity in the workplace increase, "the individual perceives that they are ignored or excluded by others", furthermore, there has not been sufficient attention on the effects of emotional exhaustion workplace ostracism and group unemployment. Therefore, this study will lead to future studies.
Keywords: Workplace, Ostracism, Emotional Exhaustion, Job Insecurity, Conservation of Resources Theory

JEL: C83, Z32, J83

\section{Introduction}

Management and hospitality in modern time have become a booming industry and one of its main focus is on the employee interaction with the industry to help provide more innovative and effective ways to improve economic growth. It is necessary for employees to be productive in their conversations and interactions with customers as well as their co-workers they engage with in their organizations or workplace which in turn is a major element in achieving efficiency and improves workplace performance. Ostracism is a major factor to look into if we are considering employee output and workplace efficiency (Liao \& Chuang, 2004; Salanova, Agut \& Peiró, 2005; Karatepe \& Olugbade, 2009).

Ostracism has numerous definitions. Numerous words have been used in different literatures to portray ostracism such as detachment, social prohibition, and dismissal, which means deserting and being unaware of what's going on (Hitlan, Cliffton \& DeSoto, 2006). Ostracism is characterized when a group of organizational employees overlooks to take activities that connect with their customers and individuals when it is ethically proper to do as such (Ferris et al., 2008). As an objective of exclusion, and employees may feel

a Res. Asst., PhD. Candidate, Eastern Mediterranean University, Faculty of Tourism, Famagusta, Northern Cyprus Via Mersin 10, Turkiye, mehmet.cizreli@emu.edu.tr (ORCID ID: 0000-0002-9884-6084)

b Asst. Prof., PhD., Cyprus Social Science University, Faculty of Human and Social Sciences, Nicosia, Northern Cyprus Via Mersin 10, Turkiye, ozlem.altun@kisbu.edu.tr (ORCID ID: 0000-0002-0830-002X)

c Res. Asst., PhD. Candidate, Eastern Mediterranean University, Faculty of Tourism, Famagusta, Northern Cyprus Via Mersin 10, Turkiye, pinarbarut@windowslive.com (ORCID ID: 0000-0002-3624-1581) 
neglected, prohibited, or overlooked by different people. Even though ostracism has a negative effect on service productivity and work engagement, in this review paper the aim will be to focus on trying to understand how this negative impact effects work engagement and service productivity using the proposed model created by putting it to test to observe what impact (WO) plays in terms of job security. The model will be tested using the (COR) conservation of resources theory which denotes that assets are important to further improve the quality of organizational workplace well-being (Hobfoll, 1989, 1998). For example, on social ostracism WO is referred to as an individual's awareness on that manner in which she or he is treated by others in the workplace (Williams, 2001, 2007), including actions like avoiding eye contact and refusing to work with the ostracized individual (Ferris et al., 2008).

COR implies that employees have a substantial value for themselves as well as things they own, therefore they tend to put in extra effort to protect the things they value. In the workplace, employees have personal values in their self-esteem and also their value workplace services such as their co-worker supports and treatments in the business (Xanthopoulou et al., 2007). The value of self-esteem of an employee helps in terms of difficulty emotionally therefore showing how well they are in a good control over their environment (Wright \& Hobfoll, 2004). Resources are naturally motivational therefore they are a part and parcel of workplace engagement and job insecurity (Hakanen et al., 2008; Karatepe \& Olugbade, 2009). From a resource-based point of view, in particular along insights from the COR feelings of $\mathrm{Jl}$ leading to exhaustion results in the loss of resources, directing the workers focus on resource conservation (Hobfoll, 2001). Without the felt job security and self-worth the individual will lack motivation for creative resourcefulness, instead engages in defending the status quo. Their position, causing resource and energy consuming. Peter (2001) described to immunize the risks of job loss the individual may extra energy in order to maintain their position by tapping into their existing resources. This however cannot be maintained over time and will lead to exhaustion of resources (Jacobson, 1991; Staufenbiel \& König, 2010; De Cuyper et al., 2012).

As an organization strives to consistently improve innovative means to enable them grow economically, ostracism can be a major hindrance and possibly lead to financial and economic loss in the organization as a whole. This review paper seeks to uncover and understand how knowledge hiding or sharing also known as ostracism can affect workplace growth. This paper focus on looking into how work ostracism leads to emotional exhaustion (EE) which leads to job insecurity (JI). The theoretical model that will be tested is shown below in Fig. 1

To address these problems, the following objectives will be our major considerations;

1. Investigate the relationship between WO and JI.

2. To explore the influence of EE on the relationship between WO and JI.

Figure 1. Theoretical Model

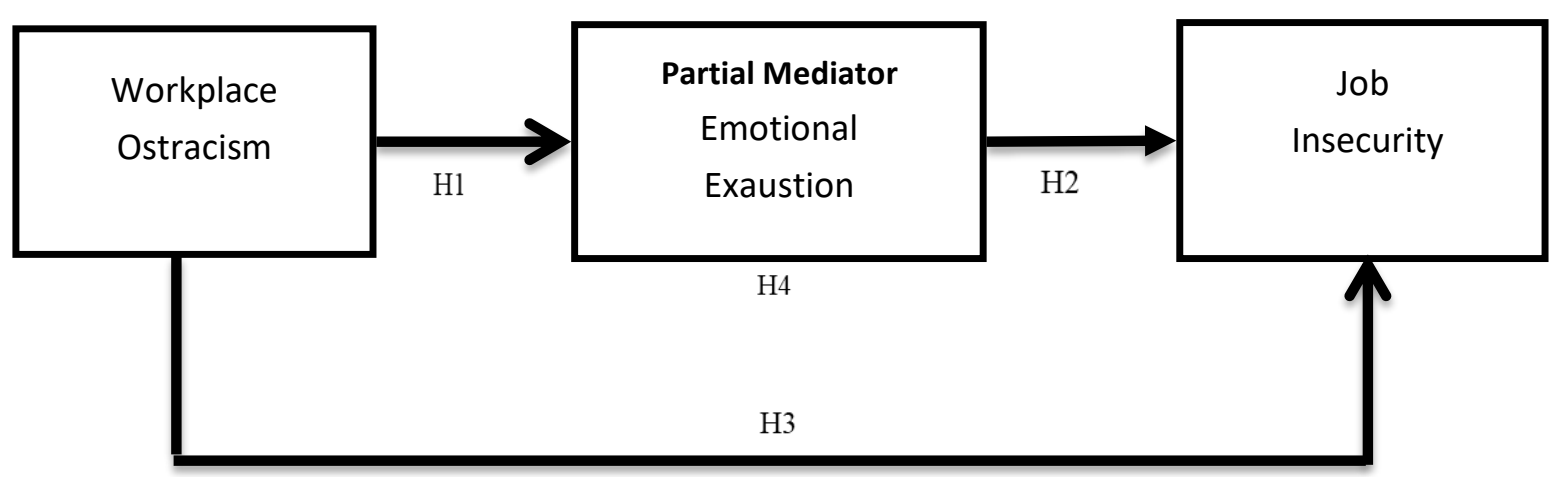


The study is organized as follows; introduction discusses the introductory path of the study and includes the aim of the study. The second part discusses the theoretical background and hypothesis. These parts give information about of Conservation of Resources Theory (COR) and hypothesis, including workplace ostracism and emotional exhaustion, emotional exhaustion and job insecurity, workplace ostracism and job insecurity and the mediating of emotional exhaustion. The third part gives information methodology, population and sample, measures and data analysis. The fourth part displayed study findings. In the last part examined conclusion.

\section{Theoretical Background and Hypothesis}

\subsection{Conservation of Resources Theory (COR)}

Concurring to COR, stress will happen if there is lost assets, an apparent danger of misfortune, or an absence of an asset increase following a speculation of assets (Hobfoll, 1989, 1998). Therefore, people endeavor to ration assets so as to manage undermining circumstances and keep themselves from negative results such as EE. When workplace organizations make an environment that empowers its representatives or employees to save or conserve resources it is noticed that employees deal with work demand effectively, therefore this help the employees with the ability to avoid negative results (Wright \& Hobfoll, 2004). On the other hand, conservation of resources (COR) hypothesis sets that asset poor representatives will endeavor to secure their remaining assets by depersonalization, lessening wok commitment, and diminishing their execution endeavors. Wright and Hobfoll (2004) clarify that depersonalization is an endeavor to limit the asset misfortunes that may come about because of communicating with customers. The conservation of resources (COR) explanation in relation to the impact of WO is related to the need-threat model of ostracism (Williams, 2001). Williams (2001), endured or consistent exposure to ostracism can affect employees and in turn result to reduction in motivation which leads to EE (Hobfoll, 1998). When individuals perceive they are ostracized from conversations and social activities and don't feel like a part of the larger group, they may discontinue their contribution to the organization, increasing a negative impact on worker contributions. If WO continues, it may cause individuals to become vulnerable, less confident, perform poorly and ultimately lead to low-value workers (Sommer \& Baumeister, 2002).

\subsection{Hypothesis}

\subsubsection{Workplace Ostracism (WO) and Emotional Exhaustion (EE)}

Ostracism at workplace is directly connected to the stressors causing diminishment of intellectual, mental, creative thought and action. It is a negative effect on their ability due to difficulty of the employees in dealing with the bad conditions that may occur, as they affect their self-confidence, control, reason and sense of belonging (Kish-Gephart et al., 2009). EE can be defined as being in a very reluctant and exhausted emotion (Wright \& Cropanzano, 1998). This is often the case when employees think that their emotional reserves are not sufficient and appropriate to manage the elements of continuous stress (Lee \& Ashforth, 1996). Freudenberger (1974) and Maslach (1976) first revealed the idea of burnout. Freudenberger (1974) describes burnout as follows; there is a decrease in energy, disinclination and exhaustion as a result of extreme and strong work, and it is much more likely to occur in people interacting with different people in intense interaction and communication. On the other hand, Maslach (1976) defines burnout as a worker's $\mathrm{EE}$, desensitized to other people; those who experience emotional exhaustion often emphasize EE as they talk about themselves or others' burnout.

Hypothesis 1: Emotional exhaustion is associated to Workplace Ostracism positively.

\subsubsection{Workplace Ostracism (WO) and Job Insecurity (JI)}

Greenhalgh \& Rosenblatt (1984) describe Jl prospects as matters concerning period of an individual's work state, outlined as the supposed quality to uphold preferred continuousness in a susceptible work environment. On the other hand, it can only be said as 'expectations of continuity in business conditions' 
(Davyet et al., 1997). When considering $\mathrm{Jl}$ relates to the workplace. Ostractisizm, structure agent, insecure employees incline responsible the organization for her/ his sufferings. Insecure employee's characteristic $\mathrm{JI}$ to the organization. As a result of employees believe the organization has broken the emotional agreement of supply secure employment circumstances, WO (Greenhalgh \& Rosenblatt, 1984; Ashford et al., 1989; Davy et al., 1997; Chiu \& Peng, 2008).

In the results of the researches, it was found that the support received from the family and friends in general were a little bit too close to the relationship between the general life dissatisfaction with the job insecurity of the employees and the physical and psychological stress and anxiety (Frese, 1999; Lim, 1996). Also, another researcher has found that external support alleviated the positive impact to job insecurity and workers' mental strain and somatic criticisms (Näswall et al., 2005). All of the results of these studies, external support's skill to diminish the loads of stressful office situations like an experience of ostracism. The reason why the perceived external support, as the reason for the investigation, is to have the effect of the exclusion of the psychological belonging needs from the elements outside the work life is the effect of the exclusion effect on the working environment and paves the way for it (Park \& Ono, 2017).

Hypothesis 2: Workplace Ostracism is related to Job Insecurity positively.

\subsubsection{Emotional Exhaustion (EE) and Job Insecurity (JI)}

Research evidence reinforces the perception that EE causes an employee to be temporarily or permanently dismissed (Öztürk et al., 2017). For example, author found a significant impacts of JI on EE in their studies on a company operating in the education segment (Tilakdharee et al., 2010). Another author was found that $\mathrm{JI}$ was significantly and positively linked to burnout in the study and they led by workers of a firm's HR (human resources) department operating in the finance area (Bosman et al., 2005). The increase in the awareness of job insecurity had a positive impact on burnout insights (Ismail, 2015). As a result of other studies with teachers in the education segment, create that JI significant and positive impact to EE (Çetin \& Turan, 2013). The results of all these studies reveal the relationship between $\mathrm{JI}$ and EE and the ensuing hypothesis is established in the light of these results.

Hypothesis 3: Employee's emotional exhaustion affects to job insecurity positively.

\subsubsection{The Mediating of Emotional Exhaustion}

EE emerged when employees started to show their emotions (Maslach, 1993). Empirical proof connecting job insecurity and geographic point ostractisizm to have been recognized (Grandey, 2003; Mulki et al., 2006; Yavas et al., 2008; Karatepe \& Aleshinloye, 2009). The high work demands in the workplaces, the employee Business Ostracism and Advanced Business Insecurity, consume emotional resources (Ladebo \& Awotunde, 2007). EE increases after the worker understands the lack of physiological and psychological resources to perform the tasks assigned to him/ her (Mulki et al., 2006). Costumercontact building employees are needed for imaginary their feelings within the technique of service distribution (e.g. Encounters with unruly customers) is subordinate in Nursing viewing emotion exhausting task, intensify heaviness and occasionally cause negative and undesirable work-related results and health matters (Chu et al., 2012; Schilpzand, Pater \& Erez, 2016). Exactly, connections between costumercontact building employees and impolite customers intensify heavily on employees' performance, that outcome in emotional exhaustion later they appreciate threats of damage, resources are unable to realize returns on their invested with resources, emotional exhaustion consecutively add increase their moods subsequent in WO also job insecurity (Hur et al., 2016; Reb et al., 2017).

Hypothesis 4: Employee Emotional Exhaustion mediates relationship between WO and Job Insecurity. 


\section{Method}

\subsection{Population \& Sample}

The scales used in the study have the necessary qualifications in terms of explaining the factors subject to analysis. The reliability and validity analyzes were performed on the scales which showed high reliability and validity in prior studies. As a result of this analysis, it was found that the reliability of the measurement tools used was quite high. The research has been applied in foreign workers working in some hotels that carry out tourism services in Northern Cyprus. From this therefore, the findings are limited to the data obtained from this group. Moreover, since the research is based on human elements and includes evaluations of employees' behaviors, the general limitations of research in the social sciences also apply to this research, and the reliability of the findings is limited to the evaluations of employees and the characteristics of the questionnaire technique used in data collection.

The aim of this study is to investigate the mediating effects EE on WO and JI using the theory of resource conservation. In accordance with the purpose of the study, we collected data by questionnaire from the hospitality sector in North Cyprus. The participants were the employees from 10 five star hotels, 5 four stars hotels and 10 other hotels. There is a total of 1535 foreign employees at all hotels in Northern Cyprus (Ministry of Tourism and Culture of Northern Cyprus, 2019). The sample size to be selected was found suitable for our sample by predicting a $5 \%$ error margin within $95 \%$ reliability limits (Naktiyok, 2019). Therefore, simple random sampling in research questionnaires were 300 questionnaires distributed, but 211 questionnaires were evaluated. A questionnaire was administered to hotel employees by emails to their human resource units and some of the questionnaires were answered by telephone correspondence, considering all confidentiality protocols. The respondents returned the completed questionnaires to the concern people in their respective units. It took about 2 months to collect this data.

\subsection{Measures}

A 10 item scale used to measure workplace ostracism; this scale was developed by Ferris et al., (2008). Responses have taken a seven point scale ranging from " 1 for never to 7 for Always" Sample questions are "Others at work shut you out of the conversation." Job insecurity measured using the Job Insecurity Scale (JIS), a scale of four items originally developed by De Witte (2000): "Chances are, I will soon lose my job". Respondents asked to rate these items on a 5-point Likert type scale, ranging from 1 ("strongly disagree") to 5 ("strongly agree"). The emotional exhaustion scale of the Utrecht Burnout Scale (the Dutch version of the MBI - General Survey will use. (Schaufeli et al., 1996; Schaufeli \& Dierendonck, 2000). Exhaustion calculated as the mean score of five items, with scores ranging from never (0) to daily (6).

\subsection{Data Analysis}

In this study, were used to SPSS and AMOS programs for data analysis. The aim why the research shows in this business dealing with pipe industrial is to control the supposed workplace ostracism of these workers who are achieved by the trustee and to control the effect of this observe on their emotional exhaustion and job insecurity levels.

\section{Findings}

Out of the 211 samples, $53.6 \%$ are male and $46.4 \%$ are female. Besides, $54.1 \%$ of the participants are single and $45.9 \%$ are married. The majority of the participants are 25 and below are old (46.9\%). The majority of the participants are undergraduate (51.7\%). Lastly, during the service period of the current hotel, it is seen that the majority of the participants have less than 1 year of service time (41.1\%). Table 1 presents the descriptive statistics of the participants. 
Table 1. Descriptive Statistics

\begin{tabular}{lcc}
\hline Demographic Questions & & \\
\hline & Frequency & Percentage \\
\hline Gender & 111 & \\
Male & 96 & 53.6 \\
Female & & 46.4 \\
Marital Status & 95 & \\
Married & 112 & 45.9 \\
Single & & 54.1 \\
Age & 97 & \\
Below 25 & 65 & 46.9 \\
26-35 & 45 & 31.4 \\
Over 35 & & 21.7 \\
Education & 51 & \\
High School or Below & 49 & 24.6 \\
Associate Degree & 107 & 23.7 \\
Undergraduate & & 51.7 \\
Length of service in current hotel & 85 & \\
Less than 1 & 70 & 41.1 \\
1-5 & 52 & 33.8 \\
6 and more & 211 & 25.1 \\
Total & & 100 \\
\hline
\end{tabular}

Table 2. CFA and EFA of Job Insecurity

\begin{tabular}{|c|c|c|c|}
\hline \multicolumn{4}{|l|}{ Items } \\
\hline \multicolumn{2}{|l|}{ Job Insecurity (JI) } & EFA & CFA \\
\hline \multicolumn{2}{|c|}{ JI1: Changes are, I will soon lose my job } & 0.956 & 0.998 \\
\hline \multicolumn{2}{|c|}{ JI2: I am sure I can keep my job. (R) } & 0.821 & 0.895 \\
\hline \multicolumn{2}{|c|}{ JI3: I feel insecure about the future of my job. } & 0.847 & 0.852 \\
\hline \multicolumn{2}{|c|}{ JI4: I think I might lose my job in the near future } & 0.922 & 0.936 \\
\hline \multirow[t]{6}{*}{ EFA } & Variance Explained (\%) & \multicolumn{2}{|c|}{88.636} \\
\hline & $\begin{array}{l}\text { Kaiser Mayer Olkin (KMO) } \\
\text { test }\end{array}$ & \multicolumn{2}{|c|}{0,768} \\
\hline & Bartlett's Test of Sphericity & \multicolumn{2}{|c|}{$X^{2}=1084.536$} \\
\hline & Chi-Square & & \\
\hline & $X^{2 / d f}$ & & \\
\hline & $\mathrm{NFI}$ & & \\
\hline \multirow[t]{3}{*}{ CFA: Model fit indices } & CFI & & \\
\hline & TLI & & \\
\hline & RMSEA & & \\
\hline Reliability Analysis & Cronbach's Alpha & & \\
\hline \multirow[t]{2}{*}{ Convergent Validity } & AVE & & \\
\hline & $\mathrm{CR}$ & & \\
\hline
\end{tabular}

According to the outcomes of the EFA and CFA of JI scale; the KMO value was 0.768 and the Cronbach alpha was value 0.959 . When KMO sample proficiency value and the Bartlett sphericity test results were considered, factor analysis was required for job insecurity scale. The total explained variance for explanatory factor was found to be $86.636 \%$. According to these results, the fact that all of the expressions have a factor load above 0.50 indicates that the substances are compatible with the structure in which they are contained. According to the results of confirmatory factor analysis, NFI, CFI, TLI and RMSE values were found to be in 
order of these values $(0.930,0.931,0.793,0.427)$. The results show that the compatibility scale values of the structural model (RMSEA, NFI, TLI and CFI) are within acceptable compliance limits. In addition, the composite reliability (CR) of all first-order constructs exceeded the threshold of 0.7 across all data sets. The results also showed a great deal of convergent validity as the Average Variance Extracted (AVE) values are well above the cutoff value of 0.5 in the job insecurity dataset. Therefore, table 2 shows the convergent validity for job insecurity (AVE: 0.787; CR: 0.936).

Moreover, in table 3 shows that emotional exhaustion which KMO was value 0.753 and the Cronbach alpha was value 0.917 . The total explained variance for explanatory factor was found to be $64.840 \%$. According to these results, the fact that all of the expressions have a factor load above 0.50 indicates that the substances are compatible with the structure in which they are contained. Only we removed seventh item with a loading of less than 0.5 . Also, the second question was removed and the " $\mathrm{e}$ " has been modified (e1-e3, e1-e4, e5-e6) as it indicates an error or unexplained variance (Pugesek et al., 2003). According to the consequences of CFA, NFI, CFI, TLI and RMSEA values were found to be in order of these values $(0.974,0.978$, $0.946,0.144)$. The results show that the compatibility scale values of the structural model (RMSEA, NFI, TLI and $\mathrm{CFI}$ ) are within acceptable values. In the table 3 also showed a great deal of convergent validity as the Average Variance Extracted (AVE) values and Composite reliability (CR) are well above the cutoff value of 0.5 in the emotional exhaustion dataset so table 3 shows the convergent validity for emotional exhaustion (AVE: 0.678; CR: 0.943).

Table 3. CFA and EFA of Emotional Exhaustion

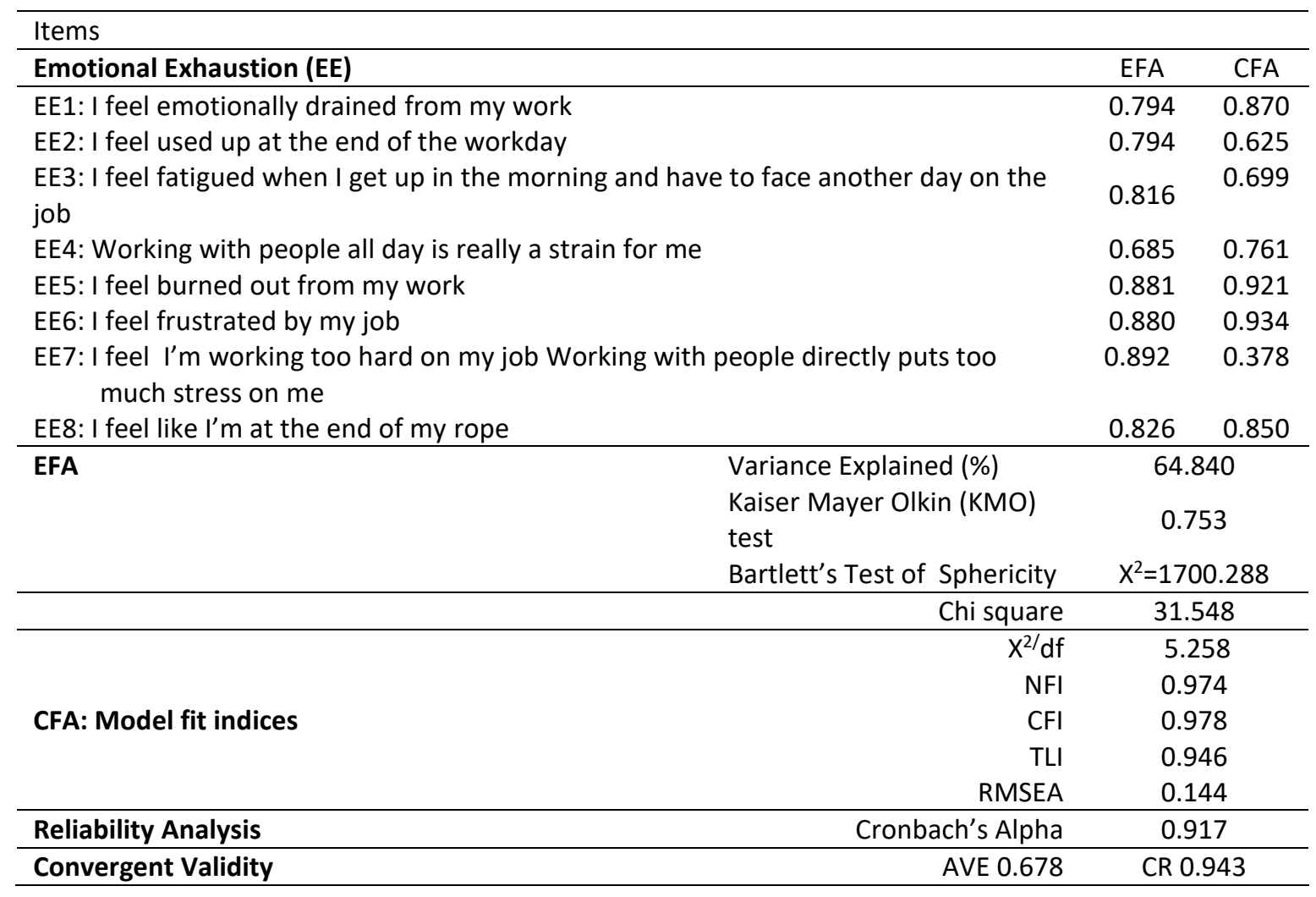

Lastly, when we examine the workplace ostracism, the results show that KMO value was 0.870 and the Cronbach alpha value was 0.978 . The total explained variance for explanatory factor was found to be $83.875 \%$. We removed any item (Q2, Q4 and Q7) to ensure compatibility. For compatibility, " $\mathrm{e}$ " has been modified (e1-e6, e5-e8, e6-e10, e6-e8) as it indicates an error or unexplained variance (Pugesek et al., 2003). According to the consequences of CFA, NFI, CFI, TLI and RMSEA values were found to be in order of these values $(0.940,0.944,0.882,0.260)$. The results show that the compatibility scale values of the structural model (RMSEA, NFI, TLI and CFI) are within acceptable values. The outcomes also showed that the composite 
reliability (CR) and Average Variance Extracted (AVE) of all first-order constructs exceeded the threshold of 0.7 across workplace ostracism data set. Furthermore, Table 4 shows the convergent validity for workplace ostracism (AVE: 0.706; CR: 0.960).

Table 4. CFA and EFA of Workplace Ostracism

\begin{tabular}{|c|c|c|c|}
\hline \multirow{2}{*}{\multicolumn{2}{|c|}{ Items }} & & \\
\hline & & EFA & CFA \\
\hline \multicolumn{2}{|c|}{ W01: Others ignored you at work when they went out for a coffee break } & 0.927 & 0.964 \\
\hline \multicolumn{2}{|c|}{ WO2: Others left the area when you entered } & 0.745 & 0.849 \\
\hline \multicolumn{2}{|c|}{ WO3: Your greeting has gone unanswered at work } & 0.892 & 0.941 \\
\hline \multicolumn{2}{|c|}{ WO4: You involuntary sat alone in a crowded lunchroom at work } & 0.665 & 0.791 \\
\hline \multicolumn{2}{|c|}{ WO5: Others avoided you at work } & 0.847 & 0.906 \\
\hline \multicolumn{2}{|c|}{ WO6: You noticed others would not look at you at work } & 0.845 & 0.915 \\
\hline \multicolumn{2}{|c|}{ WO7: Others at work shut you out of the conversation } & 0.818 & 0.897 \\
\hline \multicolumn{2}{|c|}{ WO8: Others refused to talk to you at work } & 0.903 & 0.935 \\
\hline \multicolumn{2}{|c|}{ WO9: Others at work treated you as if you weren't there } & 0.926 & 0.960 \\
\hline \multicolumn{2}{|c|}{ WO10: Others at work did not invite you or ask you if you wanted anything } & 0.820 & 0.899 \\
\hline \multirow[t]{6}{*}{ EFA } & Variance Explained (\%) & \multicolumn{2}{|c|}{83.875} \\
\hline & \multirow{2}{*}{$\begin{array}{l}\text { Kaiser Mayer Olkin (KMO) test } \\
\text { Bartlett's Test of Sphericity }\end{array}$} & \multirow{2}{*}{\multicolumn{2}{|c|}{$\begin{aligned} & 0.870 \\
X^{2}= & 3638.860\end{aligned}$}} \\
\hline & & & \\
\hline & Chi square & \multicolumn{2}{|c|}{149.648} \\
\hline & $x^{2 / d f}$ & & \\
\hline & $\mathrm{NFI}$ & \multicolumn{2}{|c|}{0.940} \\
\hline \multirow[t]{3}{*}{ CFA: Model fit indices } & $\mathrm{CFI}$ & \multicolumn{2}{|c|}{0.944} \\
\hline & TLI & \multicolumn{2}{|c|}{0.882} \\
\hline & RMSEA & \multicolumn{2}{|c|}{0.260} \\
\hline \multicolumn{2}{|r|}{ Cronbach's Alpha } & \multicolumn{2}{|c|}{0.978} \\
\hline \multicolumn{2}{|l|}{ Convergent Validity } & \multicolumn{2}{|c|}{ CR 0.960} \\
\hline
\end{tabular}

In this section, the relationship between the gender, age, working years, job insecurity, emotional exhaustion and workplace ostracism were examined. The data for the correlation relationships between variables presented in Table 5 displays that there is a significant relationship between age and gender $(p=$ $0.041, p<0.05)$. Also, working years has a substantial relationship between gender and age $(p=0.000, p$ $<0.01)$. In addition, job insecurity, emotional exhaustion and workplace ostracism variables have a significant relationship $(p=0.000, p<0.01)$. In general, a significant and positive relationship was found between all variables except age.

Table 5. Correlation Relationship between Variables

\begin{tabular}{lccccccc}
\hline \multicolumn{7}{c}{ Correlations } \\
\hline & Mean & 1 & 2 & 3 & 4 & 5 & 6 \\
\hline Gender (1) & - & 1 & & & & & \\
Age (2) & - & $-0.121^{*}$ & 1 & & & & \\
Working years (3) & - & $-0.142^{*}$ & $0.589^{* *}$ & 1 & & & \\
Job Insecurity (4) & 2.9263 & 0.099 & $-0.542^{* *}$ & $-0.416^{* *}$ & 1 & & \\
Emotional Exhaustion (5) & 3.3502 & 0.079 & $-0.745^{* *}$ & $-0.570^{* *}$ & $0.825^{* *}$ & 1 & \\
Workplace Ostracism (6) & 2.5005 & 0.113 & $-0.400^{* *}$ & $-0.434^{* *}$ & $0.606^{* *}$ & $0.604^{* *}$ & 1 \\
\hline
\end{tabular}

* Correlation is significant 0.05 level and ${ }^{* *}$ Correlation is significant 0.01 level 
The attitudes toward diversity scale was used as a covariate in each analysis, due to its significance with outcomes of interest (Becker, 2005). A series of regression analysis for each of the dependent variables was conducted to examine our hypotheses. Table 6 displays the regression results of the effect WO on JI and EE also it examines to EE on J.

First of all, according to regression analysis, WO effects on EE of employees, when beta coefficient is examined, it is seen that WO has a positive effect on EE of employees (Beta $=0,604$ ). It can be said that WO is an important predictor effect on EE of employees $\left(F(117.905)=10.858 ; R^{2}=0.365 ; p=0.00<0.05\right)$. The WO explains $36 \%$ of the EE. According to the simple regression model $(Y=-0.286+0.832 . x)$, the 1 -unit increase in WO provides an increase of 0.832 units on the EE of employees. It can be stated that these results support the hypothesis $\mathrm{H} 1$ (Workplace Ostracism is associated to emotional exhaustion positively).

Secondly, when beta coefficient is examined, it is seen that WO has a positive effect on $\mathrm{Jl}$ (Beta = $0.606)$. It can be said that there is a significant effect on JI $\left(F(119.028)=10.910 ; R^{2}=0.367 ; p=0.000<0.05\right)$. The WO explains $36 \%$ of the Jl. According to the simple regression model $(Y=-0.378+0.984 . x)$, the 1 -unit increase in WO provides an increase of 0.984 units on the Jl. It can be stated that these results support the hypothesis $\mathrm{H} 2$ (Workplace Ostracism is linked to Job Insecurity positively).

Thirdly, when beta coefficient is examined, it is seen that employee's EE has a positive effect on J (Beta $=0.825)$. It can be said that there is an important effect on JI $\left(F(437.797)=20.924 ; R^{2}=0.681 ; p=0.000\right.$ $<0.05)$. The EE explains $68 \%$ of the Jl. According to the simple regression model $(Y=0.503+0.973 . x)$, the 1 unit increase in employee's EE provide an increase of 0.973 units on the JI. It can be stated that these results support the hypothesis H3 (Employee's emotional exhaustion affects to job insecurity positively).

Table 6. Regression Analysis

\begin{tabular}{|c|c|c|c|c|c|c|}
\hline Variables & B & S.E & $\mathbf{t}$ & $\boldsymbol{\beta}$ & $\mathbf{F}$ & $\mathbf{R}^{2}$ \\
\hline \multicolumn{7}{|c|}{ Model 1: Dep. Var. WO } \\
\hline Constant & -0.378 & 0.274 & -1.379 & - & 119.028 & 0.367 \\
\hline JI & 0.984 & 0.090 & $10.910 *$ & 0.606 & & \\
\hline \multicolumn{7}{|c|}{ Model 2: Dep. Var. EE } \\
\hline Constant & 0.503 & 0.141 & $3.557^{*}$ & - & 437.797 & 0.681 \\
\hline JI & 0.973 & 0.047 & 20.924* & 0.825 & & \\
\hline \multicolumn{7}{|c|}{ Model 3: Dep. Var. WO } \\
\hline Constant & -0.286 & 0.267 & -1.070 & - & 117.905 & 0.365 \\
\hline$E E$ & 0.832 & 0.77 & $10.858 *$ & 0.604 & & \\
\hline
\end{tabular}

Note: ${ }^{*} p<0.05$

Considering the Andrew Hayes (2014) mediation model in Table 7, also specified in table 8, it was found that the indirect effect (0.437) and direct effect (0.169) of job insecurity on workplace ostracism Hypothesis 4 suggests that emotional exhaustion has a straight impact on workplace ostracism and job insecurity with employees in the hotel industry. This coefficient result of employee EE mediates relationship between WO and Jl. 
The Effect of Emotional Exhaustion on Workplace Ostracism and Job Insecurity in North Cyprus Hotel Industry

Table 7. Coefficients for the Mediating Effect

\begin{tabular}{|c|c|c|c|c|c|}
\hline Testing Paths & $B$ & SE(B) & $95 \% \mathrm{Cl}$ & $\beta$ & $\mathrm{t}$-value \\
\hline \multicolumn{6}{|c|}{$\begin{array}{l}\text { Paths c: JI } \\
R^{2}=0.367, F(1,205)=119.028, P=0.000\end{array}$} \\
\hline wo & 0.373 & 0.034 & $0.306,0.441$ & 0.606 & 10.910 \\
\hline \multicolumn{6}{|c|}{$\begin{array}{l}\text { Paths a: } E E \\
R^{2}=0.365, F(1,205)=117.905, P=0.000\end{array}$} \\
\hline wo & 0.439 & 0.040 & $0.359,0.519$ & 0.604 & 10.858 \\
\hline \multicolumn{6}{|c|}{$\begin{array}{l}\text { Path b and } c^{\prime}: \text { JI } \\
R^{2}=0.699, F(2,204)=237.151, P<0.001\end{array}$} \\
\hline WO(c') & 0.104 & 0.030 & $0.046,0.163$ & 0.169 & 3.510 \\
\hline$E E(b)$ & 0.613 & 0.041 & $0.533,0.694$ & 0.723 & 15.005 \\
\hline Total Effect (a) ${ }^{*}(\mathbf{b})$ & & & & 0.437 & \\
\hline
\end{tabular}

Table 8. Direct and Indirect Effects

\begin{tabular}{lccc}
\hline & Indirect Effect & Direct Effect & Total Effect \\
\hline $\mathrm{EE} \rightarrow \mathrm{WO}$ & - & 0.604 & 0.604 \\
$\mathrm{EE} \rightarrow \mathrm{JI}$ & - & 0.723 & 0.723 \\
$\mathrm{WO} \rightarrow \mathrm{JI}$ & 0.437 & 0.169 & 0.606 \\
\hline
\end{tabular}

Note: WO (workplace ostracism); EE (emotional exhaustion); Jl (job insecurity)

\section{Discussion and Conclusion}

Based on COR theory, this study investigates the impact of WO and the degree of conservation of resources, which, leads to EE and JI. The findings support the hypotheses and conclude that the negative impacts might be distinguished on the premise of personality. As mentioned in previous studies indicate that the workplace ostracism is detrimental to employee performance. For COR perspective, ostracism in the work environment has a direct negative psychological causal effect manifesting as emotional exhaustion, depression and work tension (Wu et al., 2012).

In this study, hypotheses have been proposed on the relationships between workplaces exclusion, $\mathrm{JI}$ and EE. Firstly, workplace ostracism found to considerably relate to EE. Almost as in previous analysis, there was a great relationship between WO and JI (Jahanzeb \& Fatima, 2018). Koay (2018) also attempts to show the effects of ostracism on EE within the workplace through the optics of COR. From the COR theory's perspective, emotional drainage and exposition of emotional capital. Secondly, employee EE found to considerably relate to $\mathrm{Jl}$. As mentioned in previous studies, $\mathrm{Jl}$ was positively linked with EE only among employees (Öztürk et al., 2017; Kerse et al., 2018). Thirdly, WO found to considerably relate to Jl. These study findings support that once staff ostracized at the work, it increases their $\mathrm{Jl}$ and ostracism at work could be a painful expertise and harmful to fret and job connected outcomes. Lastly, this study finding supported the mediatory influence of EE between $\mathrm{JI}$ and WO. EE as a mediator for reducing the negative impact of WO, support the finding of Shanghai dialect et al., (2012), in keeping with them; political skills cut back ostracismdistress relationship. Whereas this study can offer some proof for the external validity or generalizability of the examine relationships, it additionally points to the requirement for a lot of direct investigations of context by combining separate discourse options in future investigations of WO, Jl and EE (Avolio \& Gardner, 2005, Haq, 2014; Piccoli \& American state Witte, 2015). The intention of this research is to encourage future studies to lay emphasis on workplace ostracism and moreover assist management and hospitality in decreasing its prevalence and harmful effects. 


\section{End Notes}

1. This study was presented in "V. International Conference on Applied Economics and Finance \& Extended with Social Sciences (ICOAEF'19)" that organized in 8-9-10 April 2019 / Kyrenia in North Cyprus and the abstract was published in the Book of Abstract Proceeding.

\section{References}

Avolio, B. J., \& Gardner, W. L. (2005). Authentic leadership development: Getting to the root of positive forms of leadership. The Leadership Quarterly, 16(3), 315-338.

Becker, T. E. (2005). Potential problems in the statistical control of variables in organizational research: A qualitative analysis with recommendations. Organizational Research Methods, 8(3), 274-289.

Begley, P. T. (2006) Self-knowledge, capacity and sensitivity: Prerequisites to authentic leadership by school principals. Journal of Educational Administration, 44(6), 570-589.

De Cuyper, N., Mäkikangas, A., Kinnunen, U., Mauno, S., \& Witte, H. D. (2012). Cross-lagged associations between perceived external employability, job insecurity, and exhaustion: Testing gain and loss spirals according to the conservation of resources theory. Journal of Organizational Behavior, 33(6), 770-788.

De Witte, H. (2000). Work ethic and job insecurity: Assessment and consequences for wellbeing, satisfaction and performance at work. From Group to Community, 325-350.

Ferris, D. L., Brown, D. J., Berry, J. W., \& Lian, H. (2008). The development and validation of the Workplace Ostracism Scale. Journal of Applied Psychology, 93(6), 1348.

Glambek, M., Matthiesen, S. B., Hetland, J., \& Einarsen, S. (2014). Workplace bullying as an antecedent to job insecurity and intention to leave: a 6-month prospective study. Human Resource Management Journal, 24(3), 255-268.

Hakanen, J.J., Schaufeli, W.B., Ahola, K. (2008). The job demands-resources model: A three-year cross-lagged study of burnout, depression, commitment, and work engagement. Work \& Stress, 22(3), 224-241.

Haq, I. U. (2014). Workplace ostracism and job outcomes: Moderating effects of psychological capital. In Human capital without borders: Knowledge and learning for quality of life: Proceedings of the management, knowledge and learning international conference (pp. 1309-1323).

Hitlan, R. T., Cliffton, R. J., \& DeSoto, C. (2006). Perceived exclusion in the workplace: The moderating effects of gender on work-related attitudes and psychological health. North American Journal of Psychology, 8(2), 217-236.

Hobfoll, S. E. (1989). Conservation of resources: a new attempt at conceptualizing stress. American Psychologist, 44(3), 513-524.

Hobfoll, S. E. (1998). Stress, culture and community: The psychology and philosophy of stress. New York: Plenum Press.

Hobfoll, S. E. (2001). The influence of culture, community, and the nested-self in the stress process: Advancing Conservation of Resources Theory. Applied Psychology: An International Review, 50, 337-421.

Hur, W. M., Moon, T., \& Jun, J. K. (2016). The effect of workplace incivility on service employee creativity: The mediating role of emotional exhaustion and intrinsic motivation. Journal of Services Marketing, 30(3), 302-315.

Jacobson, D. (1991). The conceptual approach to job insecurity. In J. Hartley, D. Jacobson, B. Klandermans, \& T. Van Vuuren, Job insecurity: Coping with Jobs at Risk (pp. 23-39). London: Sage.

Jahanzeb, S., \& Fatima, T. (2018). How workplace ostracism influences interpersonal deviance: The mediating role of defensive silence and emotional exhaustion. Journal of Business and Psychology, 1-13.

Karatepe, O. M., \& Olugbade, O. A. (2009). The effects of job and personal resources on hotel employees' work engagement. International Journal of Hospitality Management, 28(4), 504-512.

Kerse, G., Kocak, D., \& Ozdemir, S. (2018). Does the perception of job insecurity bring emotional exhaustion? The relationship between job insecurity, affective commitment and emotional exhaustion. Business and Economics Research Journal, 9(3), 651-664.

Koay, K. Y. (2018). Workplace ostracism and cyberloafing: A moderated-mediation model. Internet Research, 28(4), 1122-1141.

Liao, H., \& Chuang, A. (2004). A multilevel investigation of factors influencing employee service performance and customer outcomes. Academy of Management Journal, 47(1), 41-58. 
The Effect of Emotional Exhaustion on Workplace Ostracism and Job Insecurity in North Cyprus Hotel Industry

Ministry of Tourism and Culture of Northern Cyprus (2019). Tourism Statistics. Retrieved Agust 18, 2019, from https://turizm.gov.ct.tr/

Naktiyok, S. (2019). Effects of organizational citizenship behaviors of hotel employees on work performance: A case study in Sivas province. Journal of Tourism and Gastronomy Studies, 7(2), 1057-1076.

Northouse, P. G. (2010). Leadership: Theory and practice (5th Ed). Thousand Oaks, CA: Sage Publications.

Öztürk, E. B., Karagonlar, G., \& Emirza, S. (2017). Relationship between job insecurity and emotional exhaustion: Moderating effects of prevention focus and affective organizational commitment. International Journal of Stress Management, 24(3), 247.

Park, J. H., \& Ono, M. (2017). Effects of workplace bullying on work engagement and health: The mediating role of job insecurity. The International Journal of Human Resource Management, 28(22), 3202-3225.

Peter, M. (2001). Occupational stress: Toward a more integrated framework. Handbook of Industrial, Work \& Organizational Psychology, Volume 2: Organizational Psychology, 93.

Piccoli, B., \& De Witte, H. (2015). Job insecurity and emotional exhaustion: Testing psychological contract breach versus distributive injustice as indicators of lack of reciprocity. Work \& Stress, 29(3), 246-263.

Pugesek, B. H., Tomer, A., \& Von Eye, A. (Eds.). (2003). Structural equation modeling: applications in ecological and evolutionary biology. New York: Cambridge University Press.

Reb, J., Narayanan, J., Chaturvedi, S., \& Ekkirala, S. (2017). The mediating role of emotional exhaustion in the relationship of mindfulness with turnover intentions and job performance. Mindfulness, 8(3), 707-716.

Salanova, M., Agut, S., \& Peiró, J. M. (2005). Linking organizational resources and work engagement to employee performance and customer loyalty: The mediating role of service climate. Journal of Applied Psychology, 90(6), 1217-1227.

Schaufeli, W. B., \& van Dierendonck, D. (2000). Utrechtse burn-out schaal: handleiding. Pearson Assessment and Information B.V, Amsterdam.

Schaufeli, W. B., Leiter, M. P. \& Maslach, C. (1996). Maslach burnout inventory - General survey (MBI-GS). In C. Maslach, S.E. Jackson, M.P. Leiter (Eds.), Maslach Burnout Inventory Manual, Consulting Psychologist Press, Palo Alto.

Sommer, K. L., \& Baumeister, R. F. (2002). Self-evaluation, persistence, and performance following implicit rejection: The role of trait self-esteem. Personality and Social Psychology Bulletin, 28(7), 926-938.

Staufenbiel, T., \& König, C. J. (2010). A model of the effects of job insecurity on performance, turnover intention and absenteeism. Journal of Occupational and Organizational Psychology, 83, 101-117.

Tian, Q., Zhang, L., \& Zou, W. (2014). Job insecurity and counterproductive behavior of casino dealers: The mediating role of affective commitment and moderating role of supervisor support. International Journal of Hospitality Management, 40, 29-36.

Williams, K. D. (2001). Ostracism: The power of silence. New York: The Guilford Press.

Williams, K. D. (2007). Ostracism. Annual Review of Psychology, 58, 425-452.

Wright, T. A., \& Hobfoll, S. E. (2004). Commitment, psychological well-being and job performance: An examination of conservation of resources (COR) theory and job burnout. Journal of Business and Management, 9(4), 389-406.

Wu, L. Z., Yim, F. H. K., Kwan, H. K., \& Zhang, X. (2012). Coping with workplace ostracism: The roles of ingratiation and political skill in employee psychological distress. Journal of Management Studies, 49(1), 178-199.

Xanthopoulou, D., Bakker, A. B., Demerouti, E., \& Schaufeli, W. B. (2007). The role of personal resources in the job demands-resources model. International Journal of Stress Management, 14(2), 21-141. 